\title{
CLASSIFICATION OF GENOTYPES OF EUCALYPTUS GLOBULUS UNDER COLD CONDITIONS USING THEIR FREE AMINO ACIDS CONTENT ON LEAVES AND REGULARIZED DISCRIMINANT ANALYSIS (RDA)
}

\author{
ROSARIO DEL P. CASTILLO ${ }^{1,2 *}$, DAVID CONTRERAS ${ }^{2}$, JAIME BAEZA $^{1,2}$, MATTHIAS OTTO $^{3}$, \\ CRISTIAN AGURTO ${ }^{1}$, JUANITA FREER ${ }^{1,2^{*}}$ \\ ${ }^{1}$ Biotechnology Center, University of Concepcion, Concepcion - Chile E-mail: - Chile \\ ${ }^{2}$ Faculty of Chemical Sciences, University of Concepcion, Concepcion-Chile. \\ ${ }^{3}$ Institute of Analytical Chemistry, TU Bergakademie Freiberg, Freiberg - Germany. \\ (Received: August 6, 2009 - Accepted: November 11, 2209)
}

\begin{abstract}
Eucalyptus globulus is an important forestry species for wood and pulp industry; however, its low cold resistance is a serious disadvantage. In this work, chemometric techniques were applied on the leaves' free amino acid content of different $E$. globulus genotypes in order to select cold tolerant genotypes. Pattern recognition methods based on regularized discriminant analysis $(R D A)$ were used to classify the genotypes by cold resistance using samples' foliar damages as reference of cold resistance. Correlation between the amino acids content and cold resistance of genotypes was determined from a partial least squares (PLS) model. $R D A$ models showed an excellent classification of genotypes ( $100 \%$ of correctly assigned genotypes in external validation), especially when $R D A$ was reduced to quadratic discriminant analysis $(Q D A)$. Arg, Tyr and Ala, showed the highest correlations with the foliar damage in the PLS model and they could be responsible to improve cold resistance to the studied genotypes.
\end{abstract}

Keywords: regularized discriminant analysis $(R D A)$, amino acids, cold resistance, Eucalyptus globulus.

\section{INTRODUCTION}

Chemometric tools, like pattern recognition and multivariate calibration methods, have applications in innumerable areas including food industry, environmental analysis, pharmaceutical industry and others. Applications in the forest industry are presently increasing, although most of the reported studies in this area are developed with the objective to quantify and predict the wood's chemical or physical properties ${ }^{1-6}$. Fewer studies using pattern recognition methods have been reported in this area ${ }^{7-11}$ where unsupervised methods, such as principal component analysis $(P C A)$, and some supervised methods like soft modeling class analogy (SIMCA) and k-nearest neighbor $(K N N)$ have been used for the classification and categorical prediction of new samples according to their wood physical properties, wood chemical composition or species classification ${ }^{7-11,8,12}$.

In this work, a statistical and a chemometric analysis using pattern recognition methods of the free amino acids of Eucalyptus globulus leaves is used to select cold tolerant genotypes of this species.

Eucalyptus globulus Labill is one of the most important forest species in Chile and the Southern Hemisphere, especially due to its extensive use in the pulp mill industry. This species is coveted for its rapid growth, high pulp yield and easy adaptation to new environments; however E. globulus has an important drawback: it is susceptible to cold conditions ${ }^{13}$. As a result, this species is often replaced by other species in low temperature regions, often reducing wood quality and pulp yield. Propagation programs are presently being developed using clonal selection of genotypes in order to increase tolerant populations. Thus, the identification of these tolerant genotypes using physiological, chemical or genetic information is needed.

Cold tolerance in plants is still a little known process; however principal theories suggest that under cold conditions the tolerant plants support ice formation in their tissues possibly accompanied by changes in the chemical composition in the cell, such as variation in the content of soluble carbohydrates, free amino acids and abscisic acid ${ }^{14}$. Application of acclimatization processes can increase cold resistance in some plants ${ }^{15}$. Cold acclimatization can be performed by expositions to low temperatures prior to colder or frost conditions.

The relationship between the cold tolerance at frost temperatures (freezing tolerance) and some chemical constituents, principally carbohydrates, has been studied in many plant species. Dionne et al. $(2001)^{16}$ demonstrated an accumulation of sucrose and high concentrations of fructans in crowns of Poa аппиа L. ecotypes after freezing temperatures exposures; however these compounds were not related with the freezing tolerance presented by the ecotypes. Some others works such as the studies over barley crops ${ }^{17}$, roots of Lonicera caerulia ${ }^{18}$, and cabbage seedlings ${ }^{19}$ show increments of sucrose, fructose and raffinose; but in transgenic Arabidopsis thaliana, the accumulation of raffinose by itself was not related to its cold tolerance ${ }^{20}$, while a positive correlation between this sugar and cold tolerance was demonstrated in Petunia hybrida ${ }^{21}$. These results indicate that the tolerance depends on the species, involves organs, and that there is no agreement with respect to the accumulation of specific sugars.

There are fewer reports in the literature about studies of amino acid content in plants under cold conditions. Most of those reports refer to the accumulation of free proline (Pro) ${ }^{14,22}$. Meza-Basso et al. (1986) ${ }^{23}$ presented evidence of a probable cryo-protector role of free Pro in Nothophagus dombeyi leaves due to its increase in fall and winter and its decrease in summer and spring. Several others works ${ }^{24-26}$ found relationships between accumulation of Pro and freezing tolerance, although other results suggest that Pro increments are due to the exposure to cold temperatures rather than an enhanced freezing tolerance ${ }^{22,27}$ ${ }^{28}$. Few studies have analyzed other amino acids ${ }^{29-31}$. Variations in the content of Glu, Gln, Ala, Asp and Asn after cold conditions have been reported in wheat ${ }^{30,31}$. Dionne et al. $(2001)^{29}$ found higher levels of Pro, Gln and Glu and greater differences of amino acid content for three ecotypes of Poa апnи L. after an acclimatization at subzero temperatures $\left(-2^{\circ} \mathrm{C}\right)$; however the amino acid levels were not related to the differential freezing tolerance of the ecotypes.

Additionally, the mentioned works used only classical statistical analysis to find the differences in the samples' amino acid content. We have previously reported a study classifying these genotypes under cold conditions in order to classify tolerant and sensible genotypes of E. globulus using regularized discriminant analysis $(R D A)$ as a supervised pattern recognition method of carbohydrate content on leaves ${ }^{12}$, where the classification did not show satisfactory results.

In this work, 28 genotypes of E. globulus were exposed to cold acclimatization treatments to improve their freezing tolerance, which was measured after frost conditions at the end of the acclimatization periods and expressed as foliar damage percentage. The leaves' amino acid content in the genotypes were quantified and used as data in a statistical and chemometric analysis to classify the genotypes according to cold resistance degree using $R D A$ as supervised pattern recognition methods. Additionally, the differentiation of the plants' acclimated or non-acclimated stages and their provenance by forest companies was studied to determine whether the species are able to increase their tolerance during the applied acclimatization periods and whether each forest company's nursery practices are important factors in the cold tolerance of the genotypes, respectively. 
In our knowledge, actually there are no studies that use a plant's amino acid content to select the genotypes according to cold resistance degree using pattern recognition methods.

\section{Theory}

\section{Pattern recognition methods}

\section{Regularized Discriminant Analysis (RDA)}

Developed by Friedman ${ }^{32}$, this technique has been used in different classifications problems ${ }^{33-37}$. Some comparisons with linear discriminant analysis $(L D A)$ and quadratic discriminant analysis $(Q D A)$, considering special cases of $R D A$ method, showed the advantages of its use, especially when illconditioned data like NIR spectra are used or when the ratio of objects to variables is not large ${ }^{37}$. In $R D A$, the classification problem can be described as follows:

The matrix $\boldsymbol{X}$, containing $n$ measurements $x_{1}, \ldots, x_{*}$, each of $p$ variables and the vector $y=\{1,2, \ldots K\}$, where $K$ is the number of groups, is used to assign objects to one of several $K$ groups. This assignment obeys a classification rule in such a way that the correct class of the object is predicted with high probability. The most often applied classification rules are based on the normal distribution of the different groups $f_{k}(x)$ for $\left.k \in\{1,2 \ldots K\}\right\}^{32}$ :

$$
f_{k}(x)=\frac{1}{(2 \pi)^{p / 2}\left|\sum_{k}\right|^{1 / 2}} \exp \left[-\frac{1}{2}\left(\boldsymbol{x}-\mu_{k}\right)^{t} \sum_{k}^{-1}\left(x-\mu_{k}\right)\right]
$$

where $\sum_{k}$ and $m_{k}$ are the class $k$ covariance matrix and mean vector, respectively, according to:

$$
\begin{gathered}
\sum_{k}=\frac{1}{n_{k}} \sum_{i=1}^{n_{k}}\left(\boldsymbol{x}_{i}-\mu_{k}\right)\left(\boldsymbol{x}_{i}-\mu_{k}\right)^{t} \\
\mu_{k}=\frac{1}{n_{k}} \sum_{i=1}^{n_{k}} \boldsymbol{x}_{i}
\end{gathered}
$$

To minimize the risk of misclassification, $\boldsymbol{x}$ is allocated to the class of maximum probability score:

$$
f_{\hat{k}}(\boldsymbol{x}) P_{\hat{k}}=\max _{k=1, \ldots, K}\left\{f_{k}(\boldsymbol{x}) P(k)\right\}
$$

Where $P(k)$ is the a-priori probability for class $k$. By assuming normal distribution in Equation (2), the expression in Equation (5) can be replaced by:

$$
d_{\hat{k}}(\boldsymbol{x})=\min _{k=1, \ldots, K} d_{k}(\boldsymbol{x})
$$

Equation (6) and equation (2) lead to the following expression:

$$
d_{k}(\boldsymbol{x})=\left(\boldsymbol{x}-\mu_{k}\right)^{t} \sum_{k}^{-1}\left(\boldsymbol{x}-\mu_{k}\right)+\ln \left|\sum_{k}\right|-2 \ln (P(k))
$$

where $d_{t}(\boldsymbol{x})$ is the discriminant score for the $k$ th class. The first term of equation (7) is the known squared Mahalanobis distance between $\boldsymbol{X}$ and $m_{k}$.

Equations (6) and (7) describe the quadratic boundaries between the classes in $Q D A$. When the class covariance matrices are presumed to be identical, the quadratic terms of equation (7) are cancelled and linear discriminant analysis $(L D A)$ is the resulting classification rule ${ }^{32}$. In the case of $L D A$, the class covariance matrices are assumed to be equal, so that a pooled covariance matrix can be used in place of equation (3).

$$
\sum_{\text {pooled }}=\frac{1}{n} \sum_{k=1}^{K} n_{k} \sum_{k}
$$

Bayes's theorem for calculation of the posterior probability $P(k \mid x)$ is used for the assignment of a new sample to class $k^{38}$.

$$
P(k \mid \boldsymbol{x})=\frac{f_{k}(\boldsymbol{x}) P(k)}{p(\boldsymbol{x})}
$$

where $P(k \mid \boldsymbol{x})$ is the a-posteriori probability for class $k, f_{k}(\boldsymbol{x})$ is the probability density function for class $k, p(x)$ is the probability density function over all classes and $P(k)$ is the previously mentioned a-priori probability for class $k$.

A sample $\boldsymbol{x}$ is then assigned to the class $k$ for which the largest posterior probability is found.

Regularization parameters Lambda (l) and Gamma (g), Risk of Misclassification

There are two parameters that have influence on the covariance matrix ${ }^{32}$. The net covariance matrix is calculated by:

$$
\hat{\sum}_{k}=\frac{\boldsymbol{S}_{\boldsymbol{k}}}{\boldsymbol{W}_{k}}=\frac{1}{\boldsymbol{W}_{k}} \cdot \sum_{k} w_{i}\left(\boldsymbol{x}_{i}-\boldsymbol{\mu}_{k}\right)\left(\boldsymbol{x}_{i}-\mu_{k}\right)^{t}
$$

Where $\boldsymbol{S}_{k}$ the unweighted estimate of the covariance matrix, $w_{i}$ is the weight assigned to each observation $i$, and $\boldsymbol{W}_{k}$ is the sum of $w_{i}$.

The $R D A$ method seeks biased estimates of the covariance matrices, $\boldsymbol{S}_{\boldsymbol{k}}$, to reduce their variances by introducing two parameters, $\lambda$ and $\gamma$, according to:

$$
\begin{gathered}
\boldsymbol{S}_{\boldsymbol{k}}(\lambda)=(1-\lambda) \boldsymbol{S}_{\boldsymbol{k}}+\lambda \boldsymbol{S} \\
\boldsymbol{W}_{\boldsymbol{k}}(\lambda)=(1-\lambda) \boldsymbol{W}_{k}+\lambda \boldsymbol{W}
\end{gathered}
$$

The first regularization parameter $\lambda$ controls the degree of shrinkage of the individual covariance matrix estimates towards the pooled estimates; the second regularization parameter $\gamma$ is responsible for weighting the Eigen values contrariwise ${ }^{32}$. Both parameters adopt values between 0 and 1 . Net covariance matrix by introduction of $l$ is calculated by:

$$
\hat{\sum}_{k}(\lambda)=\frac{S_{k}(\lambda)}{W_{k}(\lambda)}
$$

And both parameters lead to the weighted regularized covariance matrix $\hat{\sum}_{k}(\lambda, \gamma)$ :

$$
\hat{\sum}_{k}(\lambda, \gamma)=(1-\gamma) \hat{\sum}_{k}(\lambda)+\frac{\gamma}{p} \operatorname{tr}\left[\hat{\sum}_{k}(\lambda)\right] I
$$

where $t r$ characterizes the trace of a matrix and the values of $l$ and $g$ can be optimized in order to obtain the least risk of misclassification. If $\lambda=1$ and $\gamma=0, R D A$ is equal to $L D A$; and if $\lambda=0$ and $\gamma=0, R D A$ corresponds to $Q D A^{32}$.

Misclassification risk $(\operatorname{Risk}(\hat{k} \mid \boldsymbol{X}))$ of assigned sample to class $k(\hat{k})$ within matrix $\boldsymbol{X}$ can be calculated by:

$$
\operatorname{Risk}(\hat{k} \mid \boldsymbol{X})=\frac{\sum_{k=1}^{K} \operatorname{Loss}(k, \hat{k}) f_{k}(\boldsymbol{X})(P(k))}{\sum_{k=1}^{K} f_{k}(\boldsymbol{X})}
$$


Where: $\sum^{K} \operatorname{Loss}(k, \hat{k})$ is the loss matrix for the assigned sample to class $k(\hat{k})$ compared to correct classification, $f_{k}(\boldsymbol{X})$ is the probability density function for class $k$ and $P(k)$ the unconditional prior probability of observing a class $k$ member.

\section{Materials and Methods}

\section{Samples and cold acclimatization treatments}

Samples and cold acclimatization treatments were described in Castillo et. al., (2008) $)^{12}$. Briefly, 28 genotypes of E. globulus, 6-months old, (60 ramets of each genotype) with standard nutritional states from two Chilean Forest Companies (13 from ' $\mathrm{FC1}$ ' company and 15 from ' $\mathrm{FC} 2$ ' company) were used as samples. Twenty ramets of each genotype were maintained simultaneously in three cold chamber systems with different temperature profiles ${ }^{12}$ : a) cold chamber A (control chamber at $20 / 12^{\circ} \mathrm{C}$ ), b) cold chamber $\mathrm{B}$, with weak acclimatization cycle $\left(8 / 4^{\circ} \mathrm{C}\right)$, c) cold chamber $\mathrm{C}$ with strong acclimatization cycle $\left(8 / 4 /-2^{\circ} \mathrm{C}\right)$, during 31 days, with a total number of ramets of $1680(28$ genotypes $\times 20$ ramets $\times 3$ cold chambers). After that, the fourth superior pair of leaves was sampled and one pool of these for each genotype was used as sample for the chemical analysis ( 84 samples $=28$ genotypes $\times 3$ cold chambers) . After this sampling, the genotypes in the three cold chambers were maintained $12 \mathrm{~h}$ at $-6^{\circ} \mathrm{C}$ for the posterior physiological assessment of the foliar damage percentage through a backlit visual examination to determine the necrotic area on leaves. This foliar damage percentage was used as reference indicator of the plants' cold resistance degree. The classification of the genotypes according to cold resistance degree was based on two classes: class 1 (C1) consisted of tolerant genotypes (foliar damage $<50 \%$ ) and class 2 (C2) consisted of sensible genotypes (percentage of damage $\geq 50 \%$ ). Two genotypes from the cold chamber B were removed form this classification due to experimental errors, reducing the total number of samples to 82 .

\section{Extraction and Analysis of Amino Acids}

The pooled samples of each genotype were dried in an oven at $45^{\circ} \mathrm{C}$ for 24 $\mathrm{h}$. Then, the leaves were pulverized using a grinder (Moulinex A505, France) for the extraction procedure. Free amino acids were extracted using $0.25 \mathrm{~g}$ of the dried and pulverized material with $20 \mathrm{~mL}$ of distilled water at $60^{\circ} \mathrm{C}$ by shaking at $150 \mathrm{rpm}$ for $20 \mathrm{~min}$. The extracts were filtered using $0.45 \mathrm{~mm}$ hydrophilic membranes, centrifuged at $12000 \mathrm{~g}$ for $10 \mathrm{~min}$, and the aqueous phase was collected, evaporated to dryness on a rotary evaporator at $40{ }^{\circ} \mathrm{C}$ and resolubilized in $2 \mathrm{~mL}$ of nanopure water.

Asp, Glu, Asn, Ser, Thr, Arg, Ala, Tyr, Met, Trp, Val, Phe, Leu e Ile, were determined by high performance liquid chromatography (HPLC), after a precolumn derivatization procedure with o-phtalaldehyde (OPA), based in the method described by Roshius et al (2006) $)^{39}$ with some modifications. Briefly, $200 \mathrm{~mL}$ of the aqueous extract were mixed during $1 \mathrm{~min}$ in an amber vial with $1 \mathrm{~mL}$ of $0.5 \mathrm{M}$ sodium borate buffer $\mathrm{pH} 9,5$ and $400 \mathrm{~mL}$ of OPA reagent $(0.100$ $\mathrm{mg}$ OPA $+1.2 \mathrm{~mL}$ of methanol $+50 \mathrm{~mL}$ of 2-mercaptoethanol). Derivatized samples were filtered and injected into a Merck Hitachi chromatographic system equipped with an autosampler AS-4000 and a L-6200 Pump, using a LiChrospher RP- $18(5 \mathrm{~mm})$ column, maintained at $40^{\circ} \mathrm{C}$ in an oven (Eppendorf CH-430). Detection was performed using a fluorescence LaChrom F-1080 (Merck- Hitachi) detector with excitation $\left(\lambda_{\text {exc }}\right)$ and emission $\left(\gamma_{\text {em }}\right)$ wavelengths of $334 \mathrm{~nm}$ and $425 \mathrm{~nm}$, respectively.

Pro was quantified using the method of Bates ${ }^{40}$ described by Dionne et al $(2001)^{29}$ using UV-VIS spectrophotometry at $521 \mathrm{~nm}$ by means of a colorimetric reaction with ninhydrin, using $300 \mathrm{~mL}$ of the aqueous extract, $300 \mathrm{~mL}$ of glacial acetic acid and $300 \mathrm{~mL}$ of acid ninhydrine reagent $(1.25 \mathrm{~g}$ ninhydrine $+30 \mathrm{~mL}$ of glacial acetic acid and $24 \mathrm{~mL}$ of $18 \mathrm{M}$ phosphoric acid). The reaction was carried out for $1 \mathrm{~h}$ at $100^{\circ} \mathrm{C}$ with shaking at $150 \mathrm{rpm}$, and $2 \mathrm{~mL}$ of toluene were used to separate the organic phase for its colorimetric determination. An UV-VIS spectrophotometer 1650PC (Shimadzu, Kyoto, Japan) was used for this analysis.

Standard reagents of all the used amino acids (purity $>99 \%$ ) were supplied by Sigma (Darmstadt, Germany). Standard solutions with concentrations of $5,10,30,50,60,80$ and $100 \mathrm{mM}$ were used to build calibration curves. Concentrations obtained in the extracts were reported in $\mathrm{mg}$ of each amino acid per $\mathrm{g}$ of dried weight of samples ( $\mathrm{mg} \mathrm{g}^{-1}$ of dried weight). All samples were quantified in triplicate and the total number of measurements for each amino acid was 252 ( 3 extractions $\times 28$ genotypes $\times 3$ chambers). Met - Trp, and Leu -Ile were not completely resolved and they were used and reported as mixture of those amino acids.

\section{Multivariate data analysis}

Differences in the mean of amino acid contents in the genotypes were previously tested by two-way analysis of variance (ANOVA) in order to evaluate the possible influences of the forest company, the cold acclimatization treatments, and the possible effect of the interaction forest company - cold acclimatization treatment on the amino acid content. The data were logtransformed in order to produce normality and reduce heteroscedasticity. Variance homogeneity and data normal distribution were tested with Cochrane's test and normal probability plots, respectively. Post-hoc comparisons were applied using Tukey's honestly significant difference (HSD) test or the Spjotvoll-Stoline test (a generalization of Tukey's test applicable at unequal samples sizes).

In order to classify and to predict the classes of the genotypes by cold resistance degree and additionally by forest company and cold acclimatization treatments, chemometrics analysis using pattern recognition methods ( $P C A$ as exploratory analysis and $R D A$ as predictive technique) were carried out. All the replicates were included in the classification methods used. The values of foliar damage of two genotypes were removed from the data due to experimental errors and the classification according to cold resistance degree was carried out using 246 samples.

The search for a good pair of regularization parameters $l$ and $g$ in the $R D A$ classification involved an iterative process altering both parameters with constant step-widths $(0.1)$ between 0 and 1 . A surface plot with $l, g$ and $\%$ of risk calculated by Equation (14) was used for fast determination of the parameters corresponding to the least risk.

Three techniques were used to validate the predictive $R D A$ models: resubstitution using all the training set as prediction set, cross validation with the 'leave-one-out' criterion and external validation using three sets in the next (training : prediction) rates: Set $1(75: 25 \%)$, Set $2(60: 40 \%)$ and Set $3(50$ : $50 \%$ ). The results obtained in the modeling sets were evaluated using their risk of misclassification and the results obtained on the prediction set in the external validation were evaluated using the percentage of correctly assigned samples in each class.

The correlation vector from a partial least squares $(P L S)$ model was used to evaluate the correlation of each amino acid to the samples' cold resistance. $P L S$ analysis was performed using the amino acid content as $\boldsymbol{X}$ independent matrix and the foliar damage percentage as dependent variable $(\boldsymbol{Y}) . \boldsymbol{X}$ matrix was previously pre-processed by austoscaling and orthogonal signal correction (OSC) in order to exclude the variation of data not correlated with the foliar damage.

\section{Software}

ANOVA and HSD test were performed using the General Linear Model (GLM) of Statgraphics Plus (Statistical Graphics Corp. Inc) program. PCA and PLS were performed using Pirouette 4.0 (Infometrix Inc) and $R D A$ was carried out using MATLAB version 7.4.0.287 R2007a (The Math Works, Inc.) software with the routine developed by J. Friedman (1988) and reprogrammed in Matlab.

\section{RESULTS AND DISCUSSION}

\section{Statistical analysis (ANOVA)}

Table 1 summarizes the results obtained in the ANOVA applied over the amino acid content data, where most of them, excepting Arg and Ala, show differences due to the effect of the interaction forest company - cold acclimatization treatment. In the case of Ala, the genotypes show significant variation only for the cold acclimatization treatment and the effect of the forest company provenance of the samples on the amino acid content could be discarded. Arg shows significant variation in the genotypes by both, forest company and cold acclimatization treatment independently. Those results show that a variation of samples by other factor besides cold resistance degree of the genotypes exists. 
Table 1. Summary of the two-way ANOVA analysis of the amino acid content data using forest company and acclimatition treatments as independent variables. Data were log-transformed in order to reduce heteroscedasticity in the variances. $\mathbf{F}=F$ ratio, n.s. (not significant, $\boldsymbol{p} \geq 0.05$ ), significant $\boldsymbol{p}$ are denoted by: $*(0.01<\boldsymbol{p}<0.05), * *(0.001<\boldsymbol{p}<0.01), * * *(\boldsymbol{p}<0.001)$.

\begin{tabular}{|ccccccc|}
\hline & \multicolumn{5}{c|}{ Effect } \\
\cline { 2 - 7 } Amino acid & Forest Company & $\begin{array}{c}\text { Acclimatation } \\
\text { treatments }\end{array}$ & $\begin{array}{c}\text { Forest company } \\
- \text { acclimatation } \\
\text { treatments }\end{array}$ \\
\cline { 2 - 7 } & $\boldsymbol{F}$ & $\boldsymbol{p}$ & $\boldsymbol{F}$ & $\boldsymbol{p}$ & $\boldsymbol{F}$ & $\boldsymbol{p}$ \\
\hline Pro & 2.00 & n.s & 22.30 & $* * *$ & 11.61 & $* * *$ \\
Asp & 131.88 & $* * *$ & 106.20 & $* * *$ & 9.02 & $* * *$ \\
Glu & 113.13 & $* * *$ & 38.34 & $* * *$ & 11.26 & $* * *$ \\
Asn & 7.36 & $* *$ & 111.73 & $* * *$ & 14.36 & $* * *$ \\
Ser & 0.00 & n.s & 23.22 & $* * *$ & 15.19 & $* * *$ \\
Gln & 81.29 & $* * *$ & 4.54 & $*$ & 19.29 & $* * *$ \\
Thr & 1.574 & n.s & 67.88 & $* * *$ & 4.43 & $*$ \\
Arg & 11.7 & $* * *$ & 48.73 & $* * *$ & 0.47 & n.s \\
Ala & 0.25 & n.s & 420.1 & $* * *$ & 0.11 & n.s \\
Tyr & 30.6 & $* * *$ & 320.6 & $* * *$ & 5.11 & $* *$ \\
Met - Trp & 0.22 & n.s & 60.15 & $* * *$ & 10.10 & $* * *$ \\
Val & 15.6 & $* * *$ & 43.21 & $* * *$ & 11.26 & $* * *$ \\
Phe & 26.37 & $* * *$ & 265.6 & $* * *$ & 3.27 & $*$ \\
Leu - Ile & 53.5 & $* * *$ & 59.8 & $* * *$ & 9.56 & $* * *$ \\
\hline
\end{tabular}

Figure 1 shows the variation of some amino acids in each genotype under the control and acclimatization conditions. Increases in levels of Pro, Asp, Asn, Gln, Thr, Arg, Ala, Tyr and Phe were observed. In most of the cases, amino acid content increases as the cold acclimatization conditions are stronger, except in the case of Glu and Met-Trp (and in some genotypes in the case of Asp), where levels of these amino acid in cold chamber $\mathrm{C}$ genotypes are decreased.

These results are in agreement with some previously reported works, where Asp, Glu, Gln, Arg and Ala levels incremented under low non-freezing temperatures ${ }^{30,31}$, and a diminution of some of them such as Glu, when freezing temperatures were applied over the plants was observed ${ }^{23}$; however, not all these amino acids could be responsible for the increased cold resistance of the genotypes.

\section{PLS}

PLS parameters are summarized in the Table 2. The calibration and validation coefficients ( $\mathrm{rCal}$ and $\mathrm{rVal}$, respectively) show lineal correlation between the amino acids content of the samples and their foliar damage percentage by use of four principal components $\left(P C C^{\prime}\right)$. A total number of forty five samples, majority from cold chamber $\mathrm{B}$, were used in the $P L S$ model with the objective to minimize the clustering samples by the effect of the acclimatization treatments. Model was improved using OSC transformation due to the main factors even are related to the cold acclimatization treatment or forest company of the samples. Results obtained in the PLS analysis to evaluate the correlation degree of each amino acid respect to the cold resistance of the samples is shown in the Figure 2, where the correlation vector of the $P L S$ model show that Arg, followed by Tyr and Ala, are the amino acids most correlated with the foliar damage percentage in the studied genotypes (increases of these amino acids improved greater cold resistance in the samples). Inverse correlation between cold resistance and amino acid content was observed in Glu and Gln.
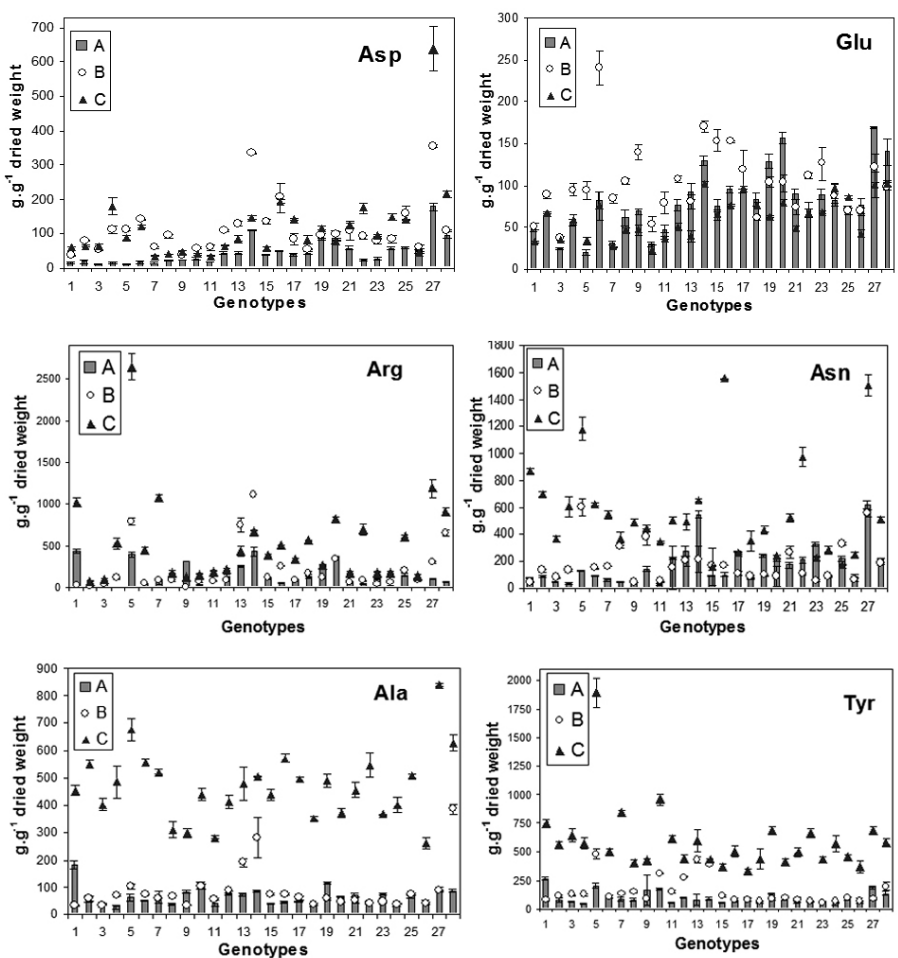

Figure 1. Amino acid concentrations in leaves of E. globulus genotypes under the following conditions: A: control conditions in cold chamber $\mathrm{A} ; \circ \mathrm{B}$ : acclimated in cold chamber $\mathrm{B}\left(8 / 4{ }^{\circ} \mathrm{C}\right)$ and $\boldsymbol{\Delta} \mathrm{C}$ : acclimated in cold chamber $\mathrm{C}\left(8 / 4 /-2^{\circ} \mathrm{C}\right)$.

Table 2. PLS parameters to predict foliar damage percentage using the amino acid content of leaves of E. globulus genotypes.

\begin{tabular}{|ccccccc|}
\hline $\begin{array}{c}\text { Number } \\
\text { of PC's } \\
(A)\end{array}$ & $\begin{array}{c}\text { Number } \\
\text { of } \\
\text { samples } \\
(\boldsymbol{n})\end{array}$ & $\begin{array}{c}\text { Cumulative } \\
\text { explained } \\
\text { variance } \\
(\%)\end{array}$ & rCal & rVal & RMSEC & RMSEV \\
\hline 4 & 45 & $75.3 \%$ & 0.9004 & 0.8300 & 6.84 & 8.33 \\
\hline
\end{tabular}

$\boldsymbol{R M S E C}$ : root mean square error of calibration calculated according to: $R M S E C=\left[\sum_{i=1}^{n_{c}}\left(y_{i}-\hat{y}_{i}\right)^{2}(n-A)\right]^{1 / 2}$

$\boldsymbol{R M S E V}$ : root mean square error of cross validation calculated according to: $R M S E V=\left[\sum_{i=1}^{n_{o}}\left(y_{i}-\hat{y}_{i}\right)^{2} / n\right]^{2}$

rCal: regression coefficient of calibration, rVal: regression coefficient of cross validation

As we have previously mentioned, in most of studies about amino acid accumulation in plants under cold conditions, principally an accumulation of free proline (Pro) is correlated with cold acclimatization or cold freezing tolerance ${ }^{24-26}$; however, in this work, free Pro levels do not show major correlation with the foliar damage of the samples. This result is in agreement with the previously mentioned works suggesting that an increase in free Pro could be merely a result of stress exposure than a cause of the enhanced freezing tolerance ${ }^{22,27,28}$. 


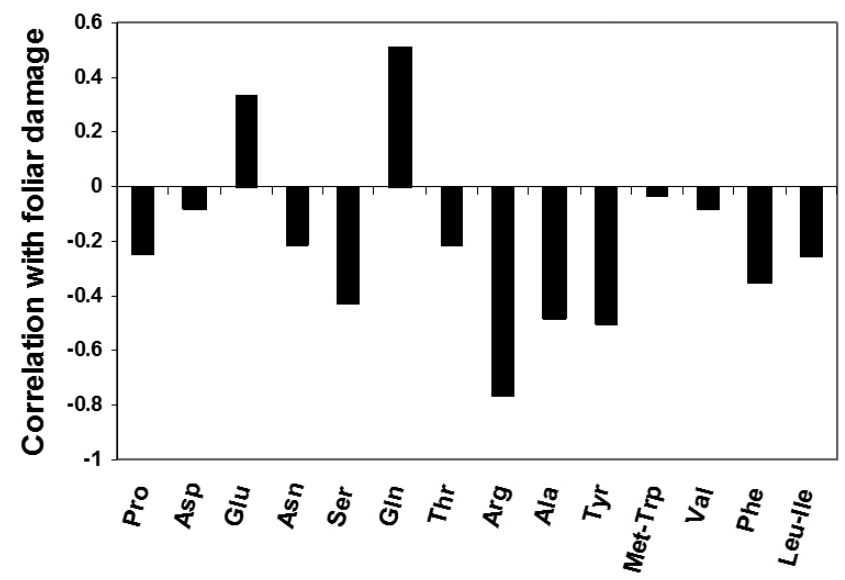

Amino acid

Figure 2. Correlation vector of each amino acid with the foliar damage percentage of the samples.

\section{Pattern recognition methods: $R D A$}

The determination of the regularization parameters in the resubstitution technique shows for all the studied classification objectives the lowest values of risk of misclassification in the range between $\mathrm{l}=0.0$ to 1.0 and $\mathrm{g}=0.0$, corresponding to the intermediates between $L D A$ and $Q D A$ methods (Figure 3ac), with a slight superiority of $Q D A$ over $L D A$ in the case of the classification by cold acclimatization treatments (Figure $3 \mathrm{~b}$ ). These regularization parameters were also tested in the validation step accompanied by the evaluation of the models' predictive ability. The results obtained in the validation of these models are summarized in the Table 3. In this table, the classification of genotypes by cold resistance degree shows that $Q D A$ is the most adequate method to correctly classify most of the sensible and tolerant genotypes in both cross validation and external validation with a high percentage of correctly assigned samples in the prediction sets, especially in the Set $1(100 \%$ of sensible and tolerant classes). As is observed in the Table 3, an increment of samples in the modeling set produces an increment in the percentage of correctly assigned samples; however an increment of the ratio of modeling : prediction samples could create a singularity problem in the application of $Q D A$ (singularity refers to the impossibility of inversion of the covariance matrix in equation 7 and is related principally to the number of samples of each class, the total number of samples and the number of variables involved in the analysis ${ }^{32}$ ). Thus, the rate 75:25 established in set 1 could be considered the best model for future predictions.

Table 3. Classification of E. globulus genotypes by cold resistance degree using RDA and leaves' amino acid content.

\begin{tabular}{|c|c|c|c|c|c|c|}
\hline \multirow{2}{*}{ Classes } & & \multicolumn{5}{|c|}{ Validation technique } \\
\hline & & Resubstitution & Cross validation & Set 1 & Set 2 & Set 3 \\
\hline \multirow{2}{*}{$\begin{array}{l}\text { Number of } \\
\text { correctly assigned } \\
\text { samples* }\end{array}$} & $\mathrm{C} 1$ & $108(86 \%)$ & $105(84 \%)$ & $29(100 \%)$ & $44(98 \%)$ & $58(97 \%)$ \\
\hline & $\mathrm{C} 2$ & $89(75 \%)$ & $84(70 \%)$ & $30(100 \%)$ & $45(85 \%)$ & $51(82 \%)$ \\
\hline \multirow{3}{*}{$\begin{array}{l}\text { Samples in the } \\
\text { prediction and } \\
\text { modeling sets }\end{array}$} & $\mathrm{SPC} 2$ & 119 & 119 & 30 & 53 & 62 \\
\hline & TSP & 244 & 244 & 59 & 98 (Set 2) & 122 \\
\hline & TSM & 244 & 244 & $185(52 \% \mathrm{C} 1,48 \% \mathrm{C} 2)$ & $146(55 \% \mathrm{C} 1,45 \% \mathrm{C} 2)$ & $122(53 \% \mathrm{C} 1,47 \% \mathrm{C} 2)$ \\
\hline
\end{tabular}

* Classified samples in prediction set. ** Risk of misclassification in the modeling set. Classes: $\mathrm{C} 1=$ sensible genotypes, $\mathrm{C} 2=$ tolerant genotypes. SPC1 $=$ Total number of $\mathrm{C} 1$ samples in the prediction set. . SPC $2=$ Total number of $\mathrm{C} 2$ samples in the prediction set. TSP $=$ Total Number of samples in the prediction set. $\mathrm{TSM}=$ Total number of samples in the modeling set. 
Unfortunately, $R D A$ classification cannot provide information about the importance of each variable for the model, but the high percentages of correctly assigned samples for the classification of genotypes by cold resistance degree in the models' external validation (100\%) indicate that it outperforms the results previously reported with the use of $R D A$ and carbohydrate content for the same data set ${ }^{12}$. This result is very important since carbohydrates are the variables usually mentioned to be correlated to the cold tolerance process, and only a few studies about the variation of amino acids have been reported in a limited number of species under cold conditions.

The results obtained in the additional classification of genotypes by cold acclimatization treatments described in the Table 4 demonstrate the superiority of $Q D A$ in the correct classification of the genotypes as well as in the cross validation and external validation. The predictive ability of these models shows a high percentage of correctly assigned samples in all the prediction sets, and especially in the sets 1 and 2 , where these percentages correspond to $100 \%$. These results suggest that the studied amino acid content can be useful for classifying genotypes not only between the genotypes acclimated in the conditions of chamber $\mathrm{C}$ with respect the others, as is observed in $P C A$, where genotypes of cold chamber A and B belong to the same cluster (plot is not shown), but also between the three cold acclimatization states in the genotypes.

Table 4. Classification of E. globulus genotypes by cold acclimatation treatment using RDA and leaves' amino acid content.

\begin{tabular}{|c|c|c|c|c|c|}
\hline \multirow{3}{*}{ Classes } & \multicolumn{5}{|c|}{ Validation techniques } \\
\hline & \multirow{2}{*}{ Resubstitution } & \multirow{2}{*}{ Cross validation } & \multicolumn{3}{|c|}{ External validation } \\
\hline & & & Set 1 & Set 2 & Set 3 \\
\hline $\mathrm{C} 1 *$ & $80(95 \%)$ & $73(87 \%)$ & $18(100 \%)$ & $33(100 \%)$ & $36(100 \%)$ \\
\hline $\mathrm{C} 2 *$ & $77(93 \%)$ & $73(88 \%)$ & $20(100 \%)$ & $35(100 \%)$ & $46(98 \%)$ \\
\hline $\mathrm{C} 3 *$ & $83(100 \%)$ & $83(100 \%)$ & $23(100 \%)$ & $32(100 \%)$ & $41(100 \%)$ \\
\hline PS C1 & 84 & 84 & 18 & 33 & 36 \\
\hline PS C2 & 83 & 83 & 20 & 35 & 47 \\
\hline PS C3 & 83 & 83 & 23 & 32 & 41 \\
\hline TPS & 250 & 250 & 61 & 100 & 124 \\
\hline TMS & 250 & 250 & $189(33.3 \%$ de cada clase $)$ & $150(33.3 \%$ de cada clase $)$ & $126(33 \%$ de cada clase $)$ \\
\hline \% Risk** & $\begin{array}{c}4.0 \\
(\lambda=0.1,0.3, \gamma=0)\end{array}$ & $\begin{array}{c}8.4 \\
(\lambda=0.0, \gamma=0.0)\end{array}$ & $\begin{array}{c}16.7 \\
(\lambda=0.0, \gamma=0.0)\end{array}$ & $\begin{array}{c}16.7 \\
(\lambda=0.0, \gamma=0.0)\end{array}$ & $\begin{array}{c}6.30 \\
(\lambda=0.0, \gamma=0.0)\end{array}$ \\
\hline
\end{tabular}

*Classified samples in prediction set. ** Risk of misclassification in the modeling set. Classes: $\mathrm{C} 1=$ genotypes in cold chamber $\mathrm{A}$ (control), $\mathrm{C} 2=$ genotypes in cold chamber $\mathrm{B}\left(8 / 4^{\circ} \mathrm{C}\right), \mathrm{C} 3=$ genotypes in cold chamber $\mathrm{C}\left(8 / 4 / 2^{\circ} \mathrm{C}\right)$. PS $\mathrm{C} 1=$ number of prediction samples of the class $\mathrm{C} 1$. PS $\mathrm{C} 2=$ number of prediction samples of the class C2. PS C3 = number of prediction samples of the class C3. TPS $=$ Total number of prediction samples. TMS $=$ Total number of samples in the modeling set.

The classification of genotypes by forest company (Table 5) showed less risk of misclassification when the analysis was carried out separately for the genotypes from each cold acclimatization system. In that case, $Q D A$ was the best technique with a risk of misclassification of $0 \%$ in resubstitution and external validation, a low risk in cross validation, and a highly predictive ability of the models, expressed by the $100 \%$ of correctly assigned samples in the prediction sets of external validation. These results show the important influence of the samples' provenances over the amino acid content in genotypes in both non-acclimated (cold chamber A) and acclimated states (cold chambers B and C). Even when the nursery practices applied by the forest companies prior to the experiments are not reported, these practices result in important differences with respect to the amino acid content between the genotypes, and thus can produce effects when a classification by cold resistance is desired. In genotypes coming from cold chamber $\mathrm{C}, L D A$ also gave high percentage of correctly assigned samples. 
Table 5. Classification of E. globulus genotypes by forest company using $R D A$ and leaves' amino acid content

\begin{tabular}{|c|c|c|c|c|c|}
\hline \multirow{2}{*}{$\begin{array}{l}\text { Validation } \\
\text { technique }\end{array}$} & \multirow{2}{*}{ Classes } & \multicolumn{4}{|c|}{ Genotypes } \\
\hline & & All data & G. in cold chamber $A$ & G. in cold chamber B & G. in cold chamber $C$ \\
\hline \multirow{4}{*}{ Resubstitution } & $\mathrm{C} 1 *$ & $108(92 \%)$ & $39(100 \%)$ & $39(64 \%)$ & $39(100 \%)$ \\
\hline & $\mathrm{C} 2 *$ & $112(84 \%)$ & $45(100 \%)$ & $44(96 \%)$ & $44(100 \%)$ \\
\hline & $\mathrm{TPS}=\mathrm{TMS}$ & 250 & 84 & 83 & 83 \\
\hline & \%Risk** & $12.7(\lambda=0.8, \gamma=0.0)$ & $0.0(\lambda=0.0-0.3,0.5-0.6, \gamma=0.0)$ & $0.0(\lambda=0.0-0.8, \gamma=0)$ & $0.0(\lambda=0.0 \gamma=0.0)$ \\
\hline \multirow{3}{*}{ Cross validation } & $\mathrm{C} 1 *$ & $101(86 \%)$ & $39(100 \%)$ & $36(92 \%)$ & $37(95 \%)$ \\
\hline & $\mathrm{C} 2 *$ & $115(86 \%)$ & $43(96 \%)$ & $43(98 \%)$ & $44(100 \%)$ \\
\hline & $\%$ Risk & $17.6(\mathrm{l}=0.8, \mathrm{~g}=0.0)$ & $2.7(\mathrm{l}=0.1, \mathrm{~g}=0.0)$ & $4.6(1=0.2-0.5, g=0)$ & $2.1(\mathrm{l}=0.2, \mathrm{~g}=0.0)$ \\
\hline \multirow{7}{*}{$\begin{array}{l}\text { External validation } \\
\quad \text { (Set 1) }\end{array}$} & $\mathrm{C} 1$ & $30(100 \%)$ & $9(100 \%)$ & $9(100 \%)$ & $12(100 \%)$ \\
\hline & $\mathrm{C} 2$ & $28(100 \%)$ & $12(100 \%)$ & $11(100 \%)$ & $8(100 \%)$ \\
\hline & PS C1 & 30 & 9 & 9 & 12 \\
\hline & PS C2 & 28 & 12 & 11 & 8 \\
\hline & TPS & 58 & 21 & 20 & 20 \\
\hline & TMS & $192(45 \% \mathrm{C} 1,55 \mathrm{C} 2 \%)$ & $63(48 \% \mathrm{C} 1,52 \% \mathrm{C} 2)$ & $63(48 \% \mathrm{C} 1,52 \% \mathrm{C} 2)$ & $63(43 \% \mathrm{C} 1,57 \% \mathrm{C} 2)$ \\
\hline & $\%$ Risk & $14.6 \%(\lambda=0.0, \gamma=0.0)$ & $0.0(\lambda=0.1-0.6, \gamma=0.0)$ & $0.0(\lambda=0.1-0.9, \gamma=0.0)$ & $0.0(\lambda=0.1-1.0, \gamma=0.0)$ \\
\hline
\end{tabular}

Table 5. (Continuation)

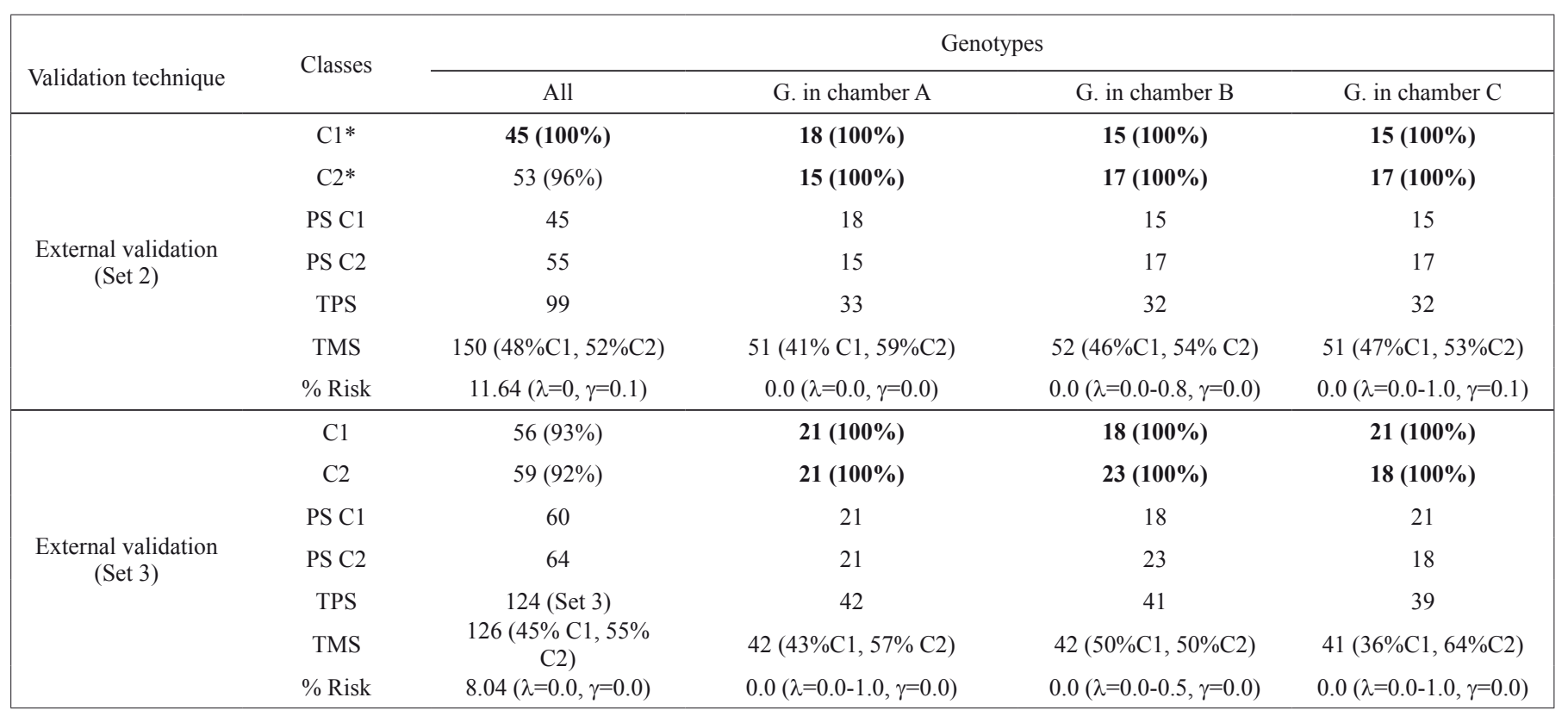

* Correctly classified samples in prediction set. ** Risk of misclassification in the modeling set. Classes: $\mathrm{C} 1=$ "FC1" genotypes, $\mathrm{C} 2=$ "FC2" genotypes. $\mathrm{PSC} 1=$ number of prediction samples of the class $\mathrm{C} 1 . \mathrm{PSC} 2=$ number of prediction samples of the class $\mathrm{C} 2 . \mathrm{TPS}=$ Total number of prediction samples. TMS $=$ Total number of samples in the modeling set.

Best results obtained using $R D A$ reduced to $Q D A$ shows that the majority of the studied classes have quadratic boundaries and that the covariance matrices of the classes are different, except in the case of the classification of the genotypes of cold chamber $\mathrm{C}$ according to Forest Company, where $L D A$ is also adequate. 
It is important to mention the advantages to use $R D A$ as predictive pattern recognition method for the evaluation of cold resistance in the genotypes, where methods based in principal component, such as SIMCA or PLS-DA (discriminant $P L S$ ) had no success due to the separation of genotypes by others factors (cold acclimatization treatment or forestry company).

\section{CONCLUSIONS}

The amino acid content evaluated in the genotypes after cold treatments was successfully used to classify the genotypes by cold resistance degree, with $100 \%$ of the samples correctly assigned in the external validation of the $R D A$ predictive model. These results show the importance of the evaluation of the amino acids levels in the response of E. globulus to cold conditions and outperform the results obtained when the carbohydrates of these same genotypes were used. The multivariate calibration of the foliar damage percentage show the highest correlation of this parameter with the amino acids Arg, Tyr and Ala and consequently these amino acids could be considered responsible for the classification of the genotypes by cold resistance degree. As we have previously mentioned, to our knowledge there are no other studies using classification methods over amino acids content to discriminate tolerant and sensible genotypes in any species, and the results of this study could be used with high accuracy in a selection program.

\section{REFERENCES}

1. Freer, J.; Ruiz, J.; Peredo, M. A.; Rodriguez, J.; Baeza, J., J. Chil. Chem. Soc. 48, 19, (2003),

2. Hauksson, J. B.; Bergqvist, G.; Bergsten, U.; Sjostrom, M.; Edlund, U., Wood Sci. Technol, 35, 475, (2001)

3. Kelley, S. S.; Rials, T. G.; Snell, R.; Groom, L. H.; Sluiter, A., Wood Sci. Technol., 38, 257, (2004)

4. Monrroy, M.; Mendonca, R.; Baeza, J.; Ruiz, J.; Ferraz, A.; Freer, J., J. Near Infrared Spectrosc. 16, 121, (2008)

5. Olsson, R. J. O.; Tomani, P.; Karlsson, M.; Joseffson, T.; Sjoberg, K.; Bjorklund, C., Tappi J. 78, 158, (1995).

6. Rials, T. G.; Kelley, S. S.; So, C. L., Wood Fiber Sci. 34, 398, (2002)

7. Alsberg, B. K.; Goodacre, R.; Rowland, J. J.; Kell, D. B., Anal. Chim. Acta, 348, 389, (1997)

8. Castillo, R.; Contreras, D.; Freer, J.; Ruíz, J.; Valenzuela, S., J. Chil. Chem. Soc, 54, 1565, (2008)

9. Gierlinger, N.; Schwanninger, M.; Wimmer, R., J. Near Infrared Spectrosc. 12, 113, (2004)

10. Michell, A. J.; Schimleck, L. R., Appita J. 51, 127, (1998)

11. Schimleck, L. R.; Michell, A. J.; Vinden, P., Appita J. 49, 319, (1996).

12. Castillo, R.; Otto, M.; Freer, J.; Valenzuela, S., J. Chemom, 22, 268, (2008)

13. Florence, R., Stan dynamic processes in: Ecology and silviculture of eucayptus forest. Australia, 1996.
14. Buchanan B; Gruissem W; R., J., Biochemistry and Molecular Biology of Plants. American Society of Plant Physiologist. John Wiley and Sons: Mariland, EE.UU, 2000.

15. Hale M., Temperature stress. In The Physiology of Plants under Stress, Orcutt, D.; Nilsen, E. John Wiley: USA, 1987.

16. Dionne, J.; Castonguay, Y.; Nadeau, P.; Desjardins, Y. Crop Sci, 41, 443, (2001)

17. Tabaei-Aghdaei, S. R.; Pearce, R. S.; Harrison, P., J. Exp. Bot, 54, 1565, (2003)

18. Imanishi, H. T.; Suzuki, T.; Masuda, K.; Harada, T., Scien. Hort. 72, 255, (1998)

19. Sasaki, H.; Ichimura, K.; Okada, K.; Oda, M., Scien.a Hort. 76, 161, (1998)

20. Zuther, E.; Buchel, K.; Hundertmark, M.; Stitt, M.; Hincha, D. K.; Heyer, A. G., FEBS Letters, 576, 169, (2004).

21. Pennycooke, J. C.; Jones, M. L.; Stushnoff, C., Plant Physiol. 133, 901, (2003)

22. Hare, P. D.; Cress, W. A.; van Staden, J., J. Exp. Bot. 50, 413, (1999)

23. Meza -Basso L. Guarda P., R. D. A. M., Phytochem. 25, 1843, (1986)

24. Dörffling K., A. M., Bradersen U., Dörffling H., Melz G., Involvement of abscisic acid and proline in cold acclimatation of winter wheat. In Plant cold hardiness, Pergamon Press: New York, 1998.

25. Koster, K. L.; Lynch, D. V., Plant Physiol. 98, 108, (1992)

26. Paquin, R.; Pelletier, G., Physiol. Vég. 19, 103, (1981)

27. Delauney, A. J.; Verma, D. P. S., Plant J. 4, 215, (1993)

28. Wanner, L. A.; Junttila, O., Plant Physiol. 120, 391, (1999)

29. Dionne, J.; Castonguay, Y.; Nadeau, P.; Desjardins, Y., Crop Sci. 41, 1862, (2001)

30. Kaldy M., F. S., J. Plant Nut. 7, 1103, (1984)

31. Naidu, B. P.; Paleg, L. G.; Aspinall, D.; Jennings, A. C.; Jones, G. P., Phytochem. 30, 407, (1991)

32. Friedman, J. H., J. Amer. Stat. Assoc. 84, 165, (1989)

33. Baldovin, A.; Wen, W.; Massart, D. L.; Turello, A., Chem. Intel. Lab. Syst. 38, 25. (1997)

34. Guo, Y. Q.; Hastie, T.; Tibshirani, R., Biostatistics, 8, 86, (2007).

35. Knauthe, B.; Steffen, S.; Barth, M.; Niewoehner, L.; Otto, M., J. Chemom. 22, 252, (2008)

36. Quinn G., K., Experimental Design and Data Analysis for Biologists. 1 ed.; Cambridge University Press: Cambridge, 2002.

37. Wu, W.; Mallet, Y.; Walczak, B.; Penninckx, W.; Massart, D. L.; Heuerding, S.; Erni, F., Anal. Chim. Acta, 329, 257, (1996)

38. Otto, M., Chemometrics. Statistics and computer application in analytical chemistry. 2nd ed.; Wiley-VCH: Weinheim, 2007.

39. Rohsius, C.; Matissek, R.; Lieberei, R., Eur. Food Res. Technol. 222, 432, (2006)

40. Bates, L., Plant Soil, 39, 205, (1973). 